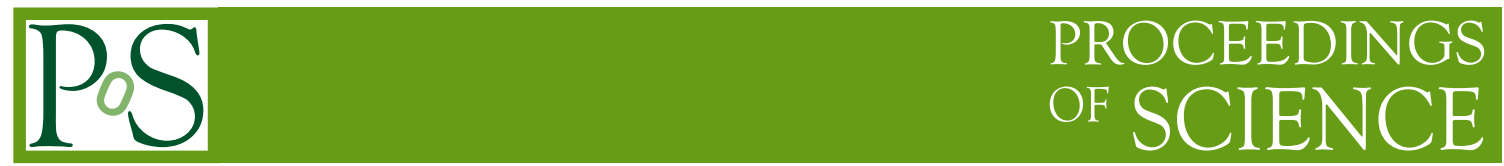

\title{
Neutrino mixing: results from accelertor experiments
}

\author{
Atsuko K. Ichikawa* \\ Kyoto University \\ E-mail: ichikawa@scphys.kyoto-u.ac.jp
}

Recent progress from accelerator-based neutrino oscillation experiments is reported. $v_{\tau}$ appearance from $v_{\mu}$ was confirmed by Super-K and OPERA. Precise measurement of $v_{\mu}$ disappearance by $\mathrm{T} 2 \mathrm{~K}$ shows value of $\theta_{23}$ still consistent with maximal mixing. $v_{e}$ appearance from $v_{\mu}$ via $\theta_{13}$ was confirmed with $7.5 \sigma$ significance by T2K. The non-zero $\theta_{13}$ discovered in 2012 implies the possibility to measure the CP-phase $\delta$, mass hierarhcy and maximal or non maximal $\theta_{23}$. T2K, NOvA and future projects will answer these questions.

The European Physical Society Conference on High Energy Physics -EPS-HEP2013 18-24 July 2013

Stockholm, Sweden

${ }^{*}$ Speaker. 


\section{Introduction}

The neutrino oscillation phenomenon tells us how the flavor eigen states and the mass eigen states mix in the lepton sector. The amount of the mixing is usually expressed in the form of a $3 \times 3$ unitary matrix:

$$
\left(\begin{array}{c}
v_{e} \\
v_{\mu} \\
v_{\tau}
\end{array}\right)=\left[\begin{array}{ccc}
U_{e 1} & U_{e 2} & U_{e 3} \\
U_{\mu 1} & U_{\mu 2} & U_{\mu 3} \\
U_{\tau 1} & U_{\tau 2} & U_{\tau 3}
\end{array}\right]\left(\begin{array}{c}
v_{1} \\
v_{2} \\
v_{3}
\end{array}\right)
$$

where the matrix is commonly parameterized as

$$
U=\left[\begin{array}{ccc}
1 & 0 & 0 \\
0 & C_{23} & S_{23} \\
0 & -S_{23} & C_{23}
\end{array}\right]\left[\begin{array}{ccc}
C_{13} & 0 & S_{13} e^{-i \delta_{C P}} \\
0 & 1 & 0 \\
-S_{13} e^{-i \delta_{C P}} & 0 & C_{13}
\end{array}\right]\left[\begin{array}{ccc}
C_{12} & S_{12} & 0 \\
-S_{12} & C_{12} & 0 \\
0 & 0 & 1
\end{array}\right]
$$

with $C_{i j}\left(S_{i j}\right)$ representing $\cos \theta_{i j}\left(\sin \theta_{i j}\right) . \theta_{i j}$ is the mixing angle between the generations $i$ and $j$. There is one irreducible phase, $\delta_{C P}$, allowed and it would induce $\mathrm{CP}$ violation in the lepton sector. With the progresses made in recent years, now all the mixing angles are known[16]:

$$
\begin{aligned}
& \theta_{12}=34^{\circ} \pm 1^{\circ} \\
& \theta_{23}=40^{\circ}+5^{\circ} /-2^{\circ} \\
& \theta_{13}= \pm 0.6^{\circ} .
\end{aligned}
$$

The remaining unknown parameter is the CP phase, $\delta_{C P}$. We also don't know the sign of $\Delta m_{32}^{2}$, which determines the mass hierarchy among neutrinos. The current value of $\theta_{23}$ is close to and consistent with the maximal mixing $\left(45^{\circ}\right)$. The targets of the coming accelerator neutrino oscillation experiments are the measurement of $\delta_{C P}$ and possibly the discovery of CP violation in the lepton sector in case $\delta_{C P} \neq 0, \pi$, determination of the mass hierarchy and to prove how close $\theta_{23}$ is to full mixing.

Long baseline neutrino experiments use $O(1) \mathrm{GeV}$ muon neutrino beams, detect them and identify the flavor at $O(100) \mathrm{km}$. The oscillation probability can be approximated by

$$
\begin{aligned}
& P\left(v_{\mu} \rightarrow v_{e}\right)=\quad 4 C_{13}^{2} S_{13}^{2} S_{23}^{2} \sin ^{2} \Phi_{31}\left(1+\frac{2 a}{\Delta m_{31}^{2}}\left(1-2 S_{13}^{2}\right)\right) \\
& +8 C_{13}^{2} S_{12} S_{13} S_{23}\left(C_{12} C_{23} \cos \delta_{C P}-S_{12} S_{13} S_{23}\right) \cos \Phi_{32} \sin \Phi_{31} \sin \Phi_{21} \\
& -8 C_{13}^{2} C_{12} C_{23} S_{12} S_{13} S_{23} \sin \delta_{C P} \sin \Phi_{32} \sin \Phi_{31} \sin \Phi_{21} \\
& +4 S_{12}^{2} C_{13}^{2}\left(C_{12}^{2} C_{23}^{2}+S_{12}^{2} S_{23}^{2} S_{13}^{2}-2 C_{12} C_{23} S_{12} S_{23} S_{13} \cos \delta_{C P}\right) \sin ^{2} \Phi_{21} \\
& -8 C_{13}^{2} S_{13}^{2} S_{23}^{2}\left(1-2 S_{13}^{2}\right) \frac{a L}{4 E} \cos \Phi_{32} \sin \Phi_{31}, \\
& P\left(v_{\mu} \rightarrow v_{\mu}\right)=1-\left(c_{13}^{4} \sin ^{2} 2 \theta_{23}+s_{23}^{2} \sin ^{2} 2 \theta_{13}\right) \sin ^{2} \Phi_{31},
\end{aligned}
$$

where $\Phi_{j i}=\Delta m_{j i}^{2} L / 4 E_{v}$. The terms which include $a \equiv 2 \sqrt{2} G_{F} n_{e} E=7.56 \times 10^{-5}\left[\mathrm{eV}^{2}\right]\left(\frac{\rho}{\left[\mathrm{g} / \mathrm{cm}^{3}\right]}\right)\left(\frac{E}{[\mathrm{GeV}]}\right)$ give rise to the matter effect, and these terms change sign like $\delta_{C P}$ for anti-neutrinos. The electron neutrino feels a different potential than muon or tau neutrinos in matter because matter contains only electrons. This difference causes the matter effect. The matter effect is approximated to first 
order in $P\left(v_{\mu} \rightarrow v_{e}\right)$ given by Eq.1.3. It is neglected together with the $\Delta m_{21}^{2}$ terms in $P\left(v_{\mu} \rightarrow v_{\mu}\right)$ given by Eq.1.4 because their contribution is very small. As can be seen in these equations, the $v_{\mu} \rightarrow v_{e}$ appearance measurement is particularly sensitive to $\sin ^{2} 2 \theta_{13}$ and $\delta_{C P}$, while the $v_{\mu} \rightarrow v_{\mu}$ disappearance measurement is particularly sensitive to $\sin ^{2} 2 \theta_{23}$ and $\Delta m_{32}^{2}$.

\section{2. $v_{\mu}$ disappearance and $v_{\mu} \rightarrow v_{\tau}$ appearance}

The most precise measurement on $\Delta m_{32}^{2}$ so far was obtained by the MINOS experiments. The MINOS experiments measured the neutrinos produced by the FNAL NuMI accelerator complex and also neutrinos produced by cosmic ray interactions in the atmosphere. It started data taking in 2005 and finished in 2012. MINOS can distinguish $v_{\mu}$ and $\overline{v_{\mu}}$ interactions by identifying the charge of the outgoing muons with a magnetized detector. Combining the data from the accelerator neutrino events and the atmospheric neutrino events, they obtained

$$
\begin{aligned}
\left|\Delta m_{\mathrm{atm}}^{2}\right| & =2.39_{-0.10}^{+0.09} \times 10^{-3} \mathrm{eV}^{2} \\
\sin ^{2} 2 \theta & =0.96_{-0.04}^{+0.04}
\end{aligned}
$$

for $v_{\mu}$ disappearance and

$$
\begin{aligned}
\left|\Delta \bar{m}_{\mathrm{atm}}^{2}\right| & =2.48_{-0.27}^{+0.22} \times 10^{-3} \mathrm{eV}^{2} \\
\sin ^{2} 2 \bar{\theta} & =0.97_{-0.08}^{+0.03}
\end{aligned}
$$

for $\overline{v_{\mu}}$ disappearance[1]. Here $\Delta m_{\mathrm{atm}}^{2}$ is an admixture of the three-flavor parameters $\Delta m_{31}^{2}$ and $\Delta m_{32}^{2}$, and it differs from $\Delta m_{32}^{2}$ by less than $2 \%$. $\theta$ is an effective two-flavor mixing angle and coincides with $\theta_{23}$ when $\theta_{13}=0$. The $\Delta \bar{m}_{\text {atm }}^{2}$ and $\bar{\theta}$ are same as $\Delta m_{\text {atm }}^{2}$ and $\theta$, but are used to indicate values measured with $\bar{v}$. They find consistent values for neutrino and antineutrino oscillation parameters, as required from CPT symmetry.

In Japan, the Tokai-to-Kamioka(T2K) long baseline experiment has been running since 2010 . Neutrinos produced at the J-PARC accelerator complex are, after traveling $295 \mathrm{~km}$, detected by the Super-Kamiokande (Super-K) water Cherenkov detector. Super-K has a good capability to distinguish sub-GeV $v_{\mu}$ and $v_{e}$ interactions by identifying an electron-like Cherenkov ring and a muon-like Cherenkov ring. They accumulated data with $6.63 \times 10^{20}$ protons on target (POT) from the accelerator, which corresponds to $8.5 \%$ of the goal statistics. They released the result of $v_{\mu}$ disappearance using data up to $3.01 \times 10^{20}$ POT. As can be seen in Eq.1.4, the $v_{\mu}$ disappearance probabilities are different depending on whether $\theta_{23}$ is smaller or larger than $45^{\circ}$ if $\theta_{13}$ is not zero, even though the values of $\sin ^{2} 2 \theta_{23}$ are the same. Since $\theta_{13}$ has now now been precisely measured by reactor experiments[2][3][4], T2K showed the allowed region of $\left|\Delta m_{32}^{2}\right|$ vs. $\sin ^{2}\left(2 \theta_{23}\right)$ for the cases of $\theta_{23}<45^{\circ}$ and of $\theta_{23}>45^{\circ}$ in Fig.1[5].

In the framework of the PMNS matrix, it is considered that atmospheric or accelerator-produced muon neutrinos mainly change to tau neutrinos. Since the threshold energy for the charged current (CC) interaction of the tau neutrino is relatively high $(\sim 3.5 \mathrm{GeV})$, we observe the 'disappearance" of muon neutrinos when the energy is not sufficient. The confirmation of the $v_{\mu} \rightarrow v_{\tau}$ oscillation is one of the important milestones for the understanding of the full picture of neutrino mixing. Super-K observed $3.8 \sigma v_{\tau}$ appearance using enhanced $\tau$-like samples from atmospheric data[7]. 


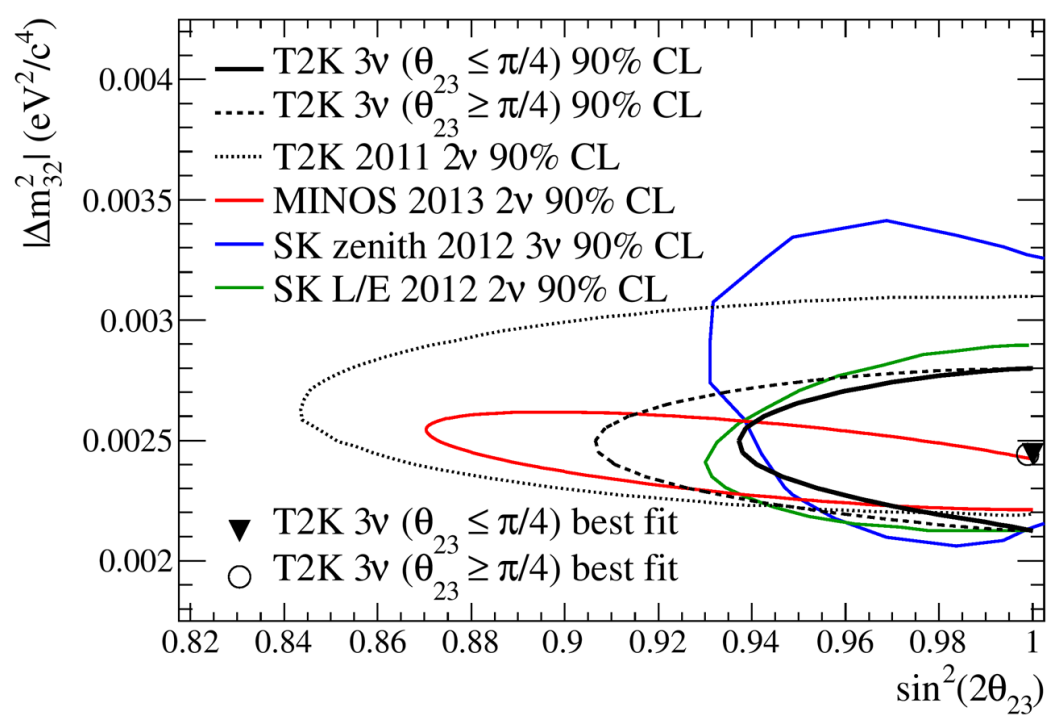

Figure 1: The allowed region of $\left|\Delta m_{32}^{2}\right|$ vs. $\sin ^{2} 2 \theta_{23}$ newly released by the T2K experiment. Those from MINOS[1] and Super-K[6] are overlaid.

Confirmation with a pure sample is also important. The OPERA experiment, using the beam from CERN SPS located $732 \mathrm{~km}$ away from the detector, identifies $\tau$ production event-by-event using nuclear emulsion. They analyzed about $60 \%$ of the data collected, and observed three $v_{\tau}$ CC interaction candidate events. This corresponds to $3.2 \sigma$ significance of $v_{\tau}$ appearance [8]. Figure 2 shows the third candidate event of $\tau$ production by a $v_{\tau}$ CC interaction, which was found in March, 2013.

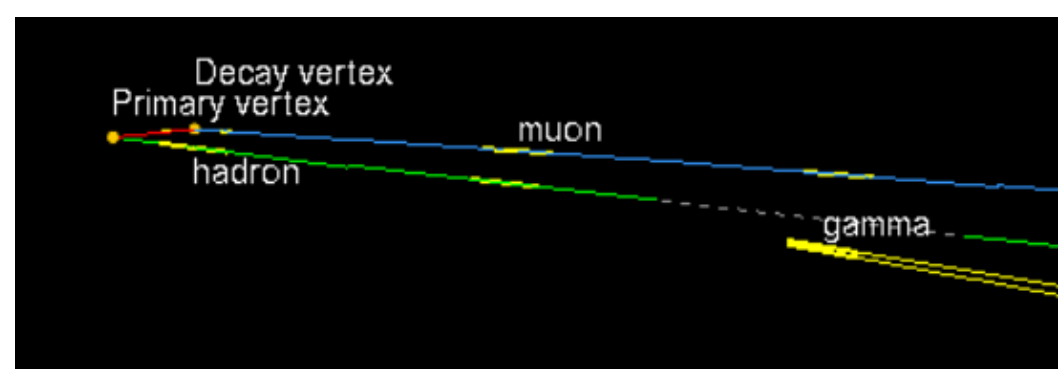

Figure 2: The third candidate event of $\tau$ production by a CC interaction of a $v_{\tau}$ oscillated from a $v_{\mu}$ observed by OPERA[8].

\section{Anomalies at small $L / E$}

The LSND (first report in 1995) and MiniBooNE experiments reported excesses of $v_{e}$ in $v_{\mu}$ beams[9][10]. Event rates measured by many reactor experiments at short distances, when compared with a newly evaluated antineutrino flux, are indicating the disappearance of $\overline{v_{e}}$, together with the results from the gallium solar neutrino calibration experiments[11]. These are not explained within the standard three-neutrino-flavor framework. Some models explain these results by 
neutrino oscillation to sterile neutrinos with a large mass squared difference $\left(\Delta m^{2}>10^{-2} \mathrm{eV}^{2}\right)$. The OPERA and ICARUS experiments have a good capability to identify $v_{e}$ interactions with nuclear emulsion or liquid Argon, and have measured it with the $v_{\mu}$ beam from CNGS. They observed no excess of $v_{e}$ interactions and set upper limits on the effective mixing angle for the $v_{\mu} \rightarrow v_{e}$ oscilla-

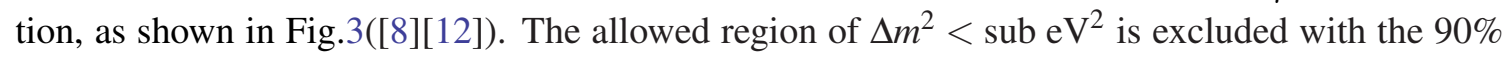
C.L. However, the larger $\Delta m^{2}$ region still remains.

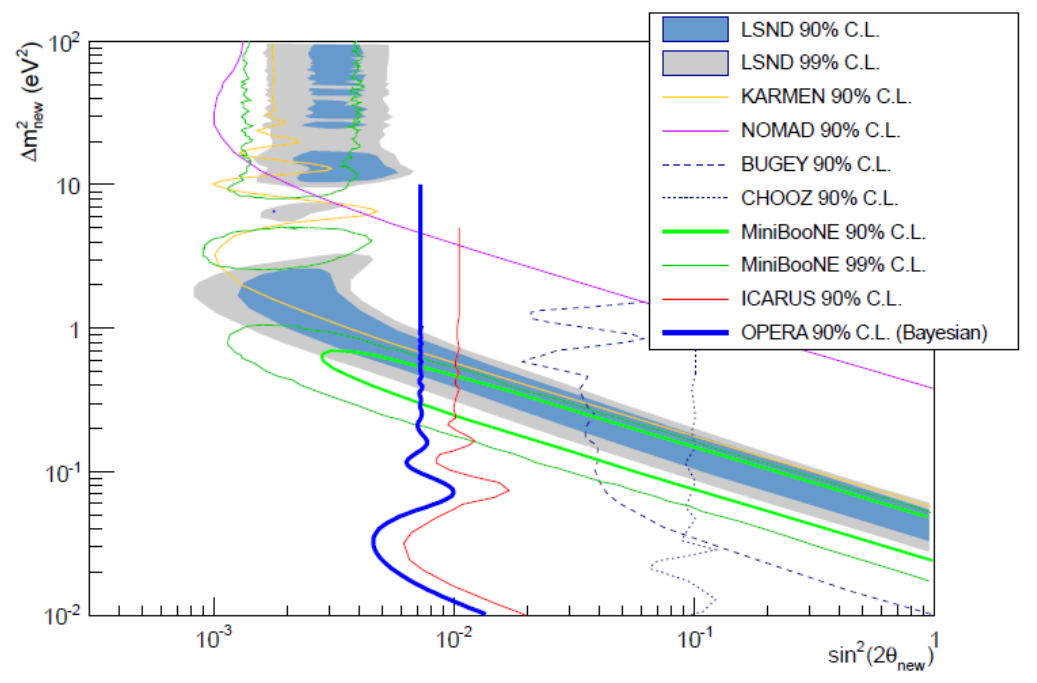

Figure 3: The upper limit on the mixing angle for $v_{\mu} \rightarrow v_{e}$ oscillation at various values of $\Delta m^{2}$ by OPERA(blue line) and ICARUS(red line). The allowed region by LSND and MiniBooNE are overlaid. Plot taken from [8]

\section{Progresses towards a precision measurement}

Neutrino oscillation experiments have entered into the era of precision measurement. There are two major sources of uncertainty to be reduced for precision measurement: the accelerator neutrino flux and the neutrino-nucleus interaction with detector materials. Progress was reported by the NA61/SHINE experiment[13] and the MINERvA experiment[14] during the EPS HEP2013 conference.

NA61/SHINE is an experiment to measure the hadron production in hadron-nucleus and nucleusnucleus collisions at the CERN SPS. It covers a wide range of the phase space of outgoing hadrons. Their measurements of the production cross sections for $\pi^{ \pm}, K^{ \pm}, p$ and $K_{S}^{0}$ for the $30 \mathrm{GeV} \mathrm{p}$-Carbon interaction were used for the prediction of the T2K neutrino flux. Thanks to these measurements, T2K achieved a highly reliable prediction of the expected far detector observables: $10 \sim 20 \%$ for the absolute flux and $\sim 2 \%$ for the extrapolation of the flux from the near detector to the far detector. NA61/SHINE released a new result with higher statistics, which will reduce the T2K flux uncertainty further. They also measured the cross sections for the NuMI target at $120 \mathrm{GeV} / c$.

Although the elementary processes of the interactions of a neutrino with a lepton or a quark are well known, many complexities arise due to the dynamical features of multi-body systems such 
as a nucleon and a nucleus. Lack of measurements causes a large uncertainty on the neutrino interaction with detector media $(10 \% \sim 100 \%)$. Contradictory observations which current models cannot explain exist. MINERvA is a neutrino scattering experiment which uses the NuMI beamline at Fermilab. They reported the result of a charged current quasi-elastic scattering measurement[14]. This interaction is the signal mode of the T2K experiment and has the advantage that the neutrino energy can be reconstructed just by the outgoing lepton momentum and angle. However, it is affected by the nuclear system. The $Q^{2}$ distribution released by MINERvA favors a transverse enhancement, which suggests a contribution from the initial-state correlations of nucleons inside the nucleus.

\section{5. $v_{e}$ appearance}

In $2011, \mathrm{~T} 2 \mathrm{~K}$ reported an indication of $v_{e}$ appearance via non-zero $\theta_{13}$ with a $2.5 \sigma$ significance, and MINOS did with a $1.7 \sigma$ significance. Then, reactor experiments reported evidence of a non-zero $\theta_{13}$ with more than $5 \sigma$ significance[2][3][4]. As can be seen in Eq.1.3, the $v_{e}$ appearance measurement would enable us to access the CP phase $\delta_{C P}$ either by comparing the $v_{\mu} \rightarrow v_{e}$ probability and the reactor $\bar{v}_{e}$ disappearance probability which is proportional to $\sin ^{2} 2 \theta_{13}$, by comparing the $v_{\mu} \rightarrow v_{e}$ and $\overline{v_{\mu}} \rightarrow \overline{v_{e}}$ probabilities, or more efficiently by comparing all of these.

MINOS placed constraints on the value of $\delta_{C P}$ by combining their measurement of $v_{e}$ appearance with the value of $\theta_{23}$ measured by $v_{\mu}$ disappearance and the value of $\sin ^{2} 2 \theta_{13}$ measured by reactor experiments as shown in Fig.4[15]. The significance is still small, but it demonstrates that we are becoming sensitive to $\delta_{C P}$.

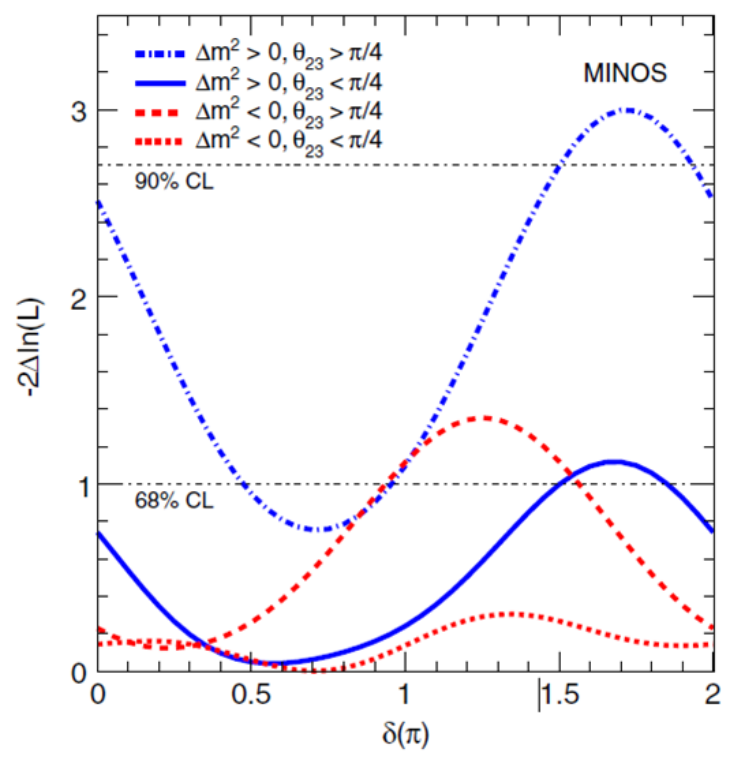

Figure 4: MINOS likelihood $L$, shown here as $-2 \Delta \ln L$, as a function of $\delta_{C P}$, assuming various values of the mass hierarchy and the octant of $\theta_{23}$.

This year, T2K updated their measurement of $v_{e}$ appearance with improved background rejection and increased statistics[5]. The background contamination was reduced by about $30 \%$ with 
a new Super-K reconstruction algorithm. The statistics were doubled: $3.01 \times 10^{20}$ POT were analyzed in 2012 and $6.39 \times 10^{20}$ POT in Apr 2013. The expected number of background events for the $v_{e}$ selection is $4.64 \pm 0.51$, while the observed number of events is 28 . Figure 5 shows the reconstructed energy of the $v_{e}$ candidate events. With this observation, $v_{e}$ appearance was confirmed with $7.5 \sigma$ significance. This is also the first time where the appearance phenomenon was confirmed with more than $5 \sigma$ significance. They fit the final state electron $(p, \theta)$ distribution to get

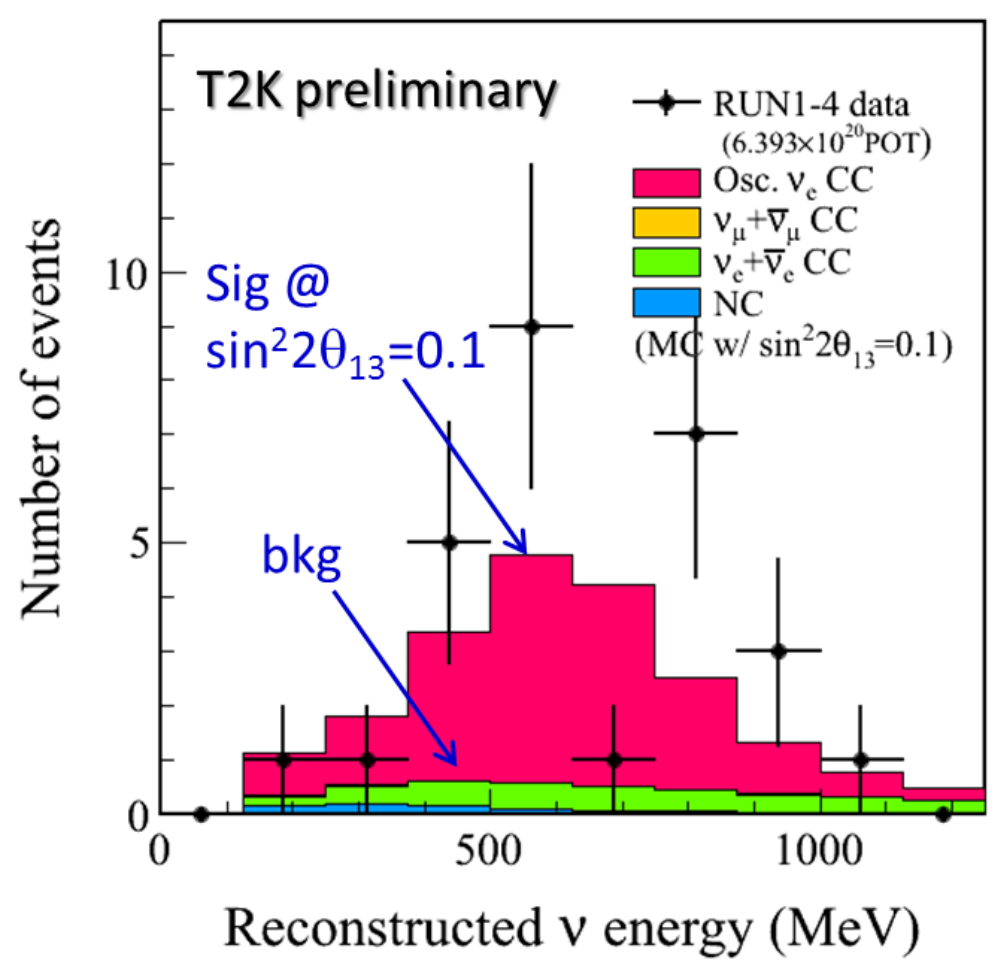

Figure 5: $\mathrm{T} 2 \mathrm{~K}$ Reconstructed neutrino energy spectrum of the $v_{e}$ candidate events[5]. Histograms show the contribution of different expected backgrounds together with the expected signal assuming $\sin ^{2} 2 \theta_{13}=$ $0.1, \delta_{C P}=0$ and the normal mass hierarchy.

$\sin ^{2} 2 \theta_{13}$. The T2K best fit value of $\sin ^{2} 2 \theta_{13}$ when $\delta_{C P}$ is fixed to 0 and $\sin ^{2} 2 \theta_{23}$ to 1 is,

$$
\begin{aligned}
& \sin ^{2} 2 \theta_{13}=0.150_{-0.034}^{+0.039} \text { for normal mass hierarchy } \\
& \sin ^{2} 2 \theta_{13}=0.182_{-0.040}^{+0.046} \text { for inverted mass hierarchy, }
\end{aligned}
$$

while the reactor results are $\sin ^{2} 2 \theta_{13}=0.098 \pm 0.013$ [16]. The allowed regions of $\sin ^{2} 2 \theta_{13}$ are shown in Fig.6 for various values of $\delta_{C P}$ and $\sin ^{2} \theta_{23}$. They are very interesting and a result with more statistics is highly desired.

\section{Future prospects}

$\mathrm{T} 2 \mathrm{~K}$ has accumulated $8.5 \%$ of the target statistics so far and will try to do a $90 \%$ confidence level measurement of $\delta_{C P}$ and precise measurements of $\theta_{23}$ and $\Delta m_{32}^{2}$. In the US, the NOvA 

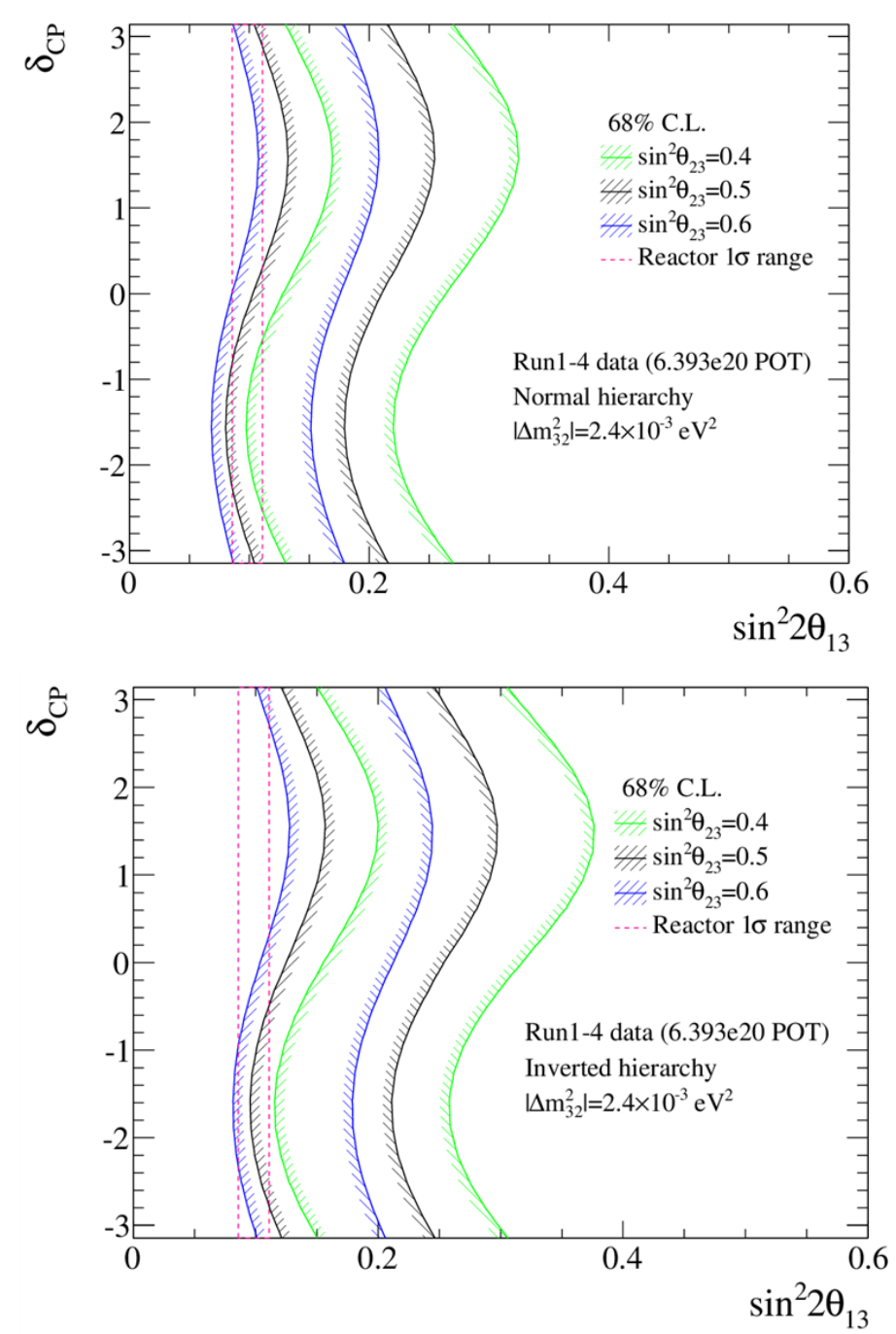

Figure 6: The allowed region of $\sin ^{2} 2 \theta_{13}$ for various values of $\delta_{C P}$ and $\sin ^{2} \theta_{23}$ [5]. The top(bottom) panel shows the $68 \% \mathrm{CL}$ region assuming the normal (inverted) mass hierarchy. The PDG reactor $1 \sigma$ range is overlaid[16].

experiment was started beam commissioning. NOvA uses muon neutrinos from the NuMI beamline at Fermilab and detects them in Minnesota, $819 \mathrm{~km}$ away from the beamline. Their detector is made of liquid scintillator. About 40\% of the detector was filled at the time of EPS HEP 2013[17], and filling is expected to be completed by April/May 2014. Since NOvA's baseline is 2.8 times longer than that of $\mathrm{T} 2 \mathrm{~K}$, their neutrino energy at the oscillation peak and accordingly the size of the matter effect are larger by roughly same amount. Therefore, they have higher sensitivity to resolve the mass hierarchy. Furthermore, it is expected that sensitivities for $\mathrm{CP}$ violation and the mass hierarchy are significantly increased by combining the results from T2K and NOvA, two experiments at different baseline lengths. We may see the answer for the mass hierarchy and an indication of the $\mathrm{CP}$ violation at some point from these two experiments.

In the longer term, it is desirable to explore $\mathrm{CP}$ violation and the mass hierarchy with $>3 \sigma$ 
sensitivity. Many projects are proposed to achieve these goals[18]:

- LBNE, $L=1,300 \mathrm{~km}$ (Fermilab to SANFORD LAB, South Dakota), liquid Argon detector[19],

- Hyper-Kamiokande, $L=295$ km (J-PARC to Kamioka), water Cherenkov detector[20]

- LAGUNA-LBNO, $L=2,300 \mathrm{~km}$ (CERN to Pyhasalmi), liquid Argon detector and/or liquid scintillator detector[18],

- EUROSB, $L=540 \mathrm{~km}$, (European Spallation Source at Lund to Garpenberg), water Cherenkov detector (with many other options for far cite locations and detectors)[21].

\section{Summary}

Rapid progress was seen in the field of neutrino oscillation this year. $v_{\tau}$ appearance from $v_{\mu}$ was confirmed by Super-K and OPERA. Precise measurement of $v_{\mu}$ disappearance by T2K shows a value of $\theta_{23}$ which is still consistent with maximal mixing. $v_{e}$ appearance from $v_{\mu}$ via $\theta_{13}$ was confirmed with $7.5 \sigma$ significance by T2K. The discovery of a non-zero $\theta_{13}$ in 2012 is not the end of the story, but is the start of a new quest: a search for $\mathrm{CP} \delta$, the mass hierarchy and maximal or non maximal $\theta_{23}$. We may soon start seeing hints about $\mathrm{CP} \delta$ from T2K and NOvA experiments, and future projects will firmly answer these questions.

\section{References}

[1] P. Adamson et al. [MINOS Collaboration], Phys. Rev. Lett. 110, 251801 (2013)

[2] F. P. An et al. [DAYA-BAY Collaboration], Phys. Rev. Lett. 108, 171803 (2012)

[3] J. K. Ahn et al. [RENO Collaboration], Phys. Rev. Lett. 108, 191802 (2012)

[4] Y. Abe et al. [DOUBLE-CHOOZ Collaboration], Phys. Rev. Lett. 108, 131801 (2012)

[5] M. Wilking for the T2K collaboration, 'New Results from the T2K Experiment: Observation of $v_{e}$ Appearance from a $v_{\mu}$ Beam", talk given at EPS HEP 2013.

[6] Y. Itow, 'Atmospheric neutrinos Results from running experiments ' talk given at neutrino2012.

[7] K. Abe et al. [Super-Kamiokande Collaboration], Phys. Rev. Lett. 110, 181802 (2013)

[8] A. Pastore for the OPERA collaboration, 'Recent results of the OPERA neutrino experiment', talk given at EPS HEP 2013.

[9] A. Aguilar it et al., Phys. Rev. D 64, 112007 (2001) and references cited there.

[10] A. A. Aguilar-Arevalo et al. [MiniBooNE Collaboration], Phys. Rev. Lett. 110, 161801 (2013)

[11] G. Mention it et al., Phys. Rev. D 83, 073006 (2011)

[12] E. Segreto [ICARUS Collaboration], J. Phys. Conf. Ser. 447, 012064 (2013).

[13] A. Korzenev for the NA61/SHINE collaboration, 'Hadron production measurements from NA61/SHINE', talk given at EPS HEP 2013.

[14] C. Patric for the MINERvA collaboration, 'Charged Current Quasi-Elastic Scattering at MINERvA', talk given at EPS HEP 2013. 
[15] P. Adamson et al. [MINOS Collaboration], Phys. Rev. Lett. 110, 171801 (2013)

[16] J. Beringer et al. [Particle Data Group], Phys. Rev. D86, 010001 (2012)

[17] A. Waldron for the NOvA experiment, 'The NOvA Experiment', talk given at EPS HEP 2013.

[18] A. Rubbia, 'Neutrino program: the future', talk given at EPS HEP2013

[19] E. Worcester for the LBNE collaboration, 'LBNE IN THE PRECISION ERA OF NEUTRINO OSCILLATION', talk given at EPS HEP 2013.

[20] Hide Tanaka, 'Hyper-Kamiokande Project', talk given at EPS HEP 2013.

[21] T. Ekelof, 'A lepton CP violation discovery experiment using a unique neutrino Super Beam', talk given at EPS HEP 2013. 